\title{
Entre asymétrie d'information et incertitude partagée. Analyse des systèmes de certification biologique pour le marché domestique kenyan
}

Between information asymmetry and shared uncertainty, an analysis of organic certification systems for the Kenyan domestic market

Chloé Tankam, Dominique Vollet et Olivier Aznar

\section{(2) OpenEdition \\ Journals}

Édition électronique

URL : http://journals.openedition.org/economierurale/6982

DOI : $10.4000 /$ economierurale.6982

ISSN : 2105-2581

\section{Éditeur}

Société Française d'Économie Rurale (SFER)

Édition imprimée

Date de publication : 30 septembre 2019

Pagination : 63-81

ISSN : 0013-0559

\section{Référence électronique}

Chloé Tankam, Dominique Vollet et Olivier Aznar, « Entre asymétrie d'information et incertitude partagée. Analyse des systèmes de certification biologique pour le marché domestique kenyan », Économie rurale [En ligne], 369 | Juillet-septembre, mis en ligne le 01 janvier 2021, consulté le 06 janvier 2021. URL : http://journals.openedition.org/economierurale/6982 ; DOI : https://doi.org/ 10.4000/economierurale.6982 


\title{
Entre asymétrie d'information et incertitude partagée
}

\section{Analyse des systèmes de certification biologique pour le marché domestique kenyan}

\author{
Chloé TANKAM • Dominique VOLLET • Olivier AZNAR • Université Clermont Auvergne, \\ AgroParisTech, Inra, Irstea, VetAgro Sup, Territoires, Clermont-Ferrand \\ chloe.tankam@agroparistech.fr ; dominique.vollet@irstea.fr ; olivier.aznar@vetagro-sup.fr
}

Les marchés de produits agricoles biologiques kenyans sont confrontés à un double enjeu d'asymétrie d'information et d'incertitude partagée. Plusieurs systèmes de certification ont émergé, posant la question de leur contribution à la pérennité de ces marchés. Les auteurs proposent de les comparer à partir d'une grille d'analyse originale complétant les apports de l'économie de I'information par le concept d'incertitude partagée. Cette grille a été appliquée à partir d'entretiens semi-directifs menés auprès des différents acteurs des filières. Le système participatif de garantie étudié ici, principal dispositif de contrôle conservé par les producteurs, s'avère le moins efficace dans la prise en compte de l'asymétrie informationnelle et de l'incertitude partagée, pourtant renforcée sur ces marchés émergents et dans une phase de transition entre dispositifs.

MOTS-CLÉS : certification, système participatif de garantie, agriculture biologique, économie de la qualité, marchés domestiques, Kenya

\section{Between information asymmetry and shared uncertainty, an analysis of organic certification systems for the Kenyan domestic market}

The Kenyan organic agricultural product markets face two main challenges: information asymmetry and shared uncertainty among partners. In order to analyze the stability of these markets, we compare different certification and control systems. Thus, we have built a specific analytical framework regarding the determinants of choices, which we have applied based on semi-structured interviews conducted with stakeholders in the sectors. The major control mechanism, Participatory Guarantee Systems (PGSs), is the least effective in taking account of information asymmetry and shared uncertainty, which has increased in these emerging markets and in a period of transition between mechanisms. (JEL: D80, Q01)

KEYWORDS: certification, participatory guarantee system, organic farming, Kenya, economy of quality, domestic markets

L 'agriculture biologique n'est plus l'apa$44 \%$ des producteurs biologiques dans $44 \%$ des producteurs biologiques dans le monde se situaient en Asie, en Afrique et en Amérique latine, avec en tête l'Inde et l'Ouganda (Willer et al., 2018). La superficie agricole convertie et certifiée est passée en Afrique de près d'un million d'hectares en 2001 à 1,8 million en 2016
(Willer et al., 2018). Toutefois, l'essentiel de ces productions reste des cultures de rente, exportées dans les pays développés et donc soumises à des certifications internationales difficiles d'accès pour les petits producteurs. Considérant la contribution de l'agriculture biologique à la lutte contre la pauvreté, la Fédération internationale pour l'agriculture biologique (IFOAM) 
a encouragé le développement de modes de contrôle alternatifs et notamment les Systèmes participatifs de garantie (SPG). Les SPG sont définis comme «des systèmes d'assurance $[\ldots]$ qui certifient les producteurs sur la base d'une participation active des acteurs concernés. [Ils] sont construits sur une base de confiance, de réseaux et d'échanges de connaissances » (May, 2008). Pionnière sur le continent, l'Afrique de l'Est a mis en place un label biologique est-africain en 2012, autorisant les certifications par tiers via des certificateurs locaux et les SPG.

Au Kenya, des Organisations non gouvernementales $(\mathrm{ONG})$ ont fait le choix de développer des marchés biologiques locaux qui sont passés de trois en 2006 à sept en 2012. Il s'agit principalement de marchés de producteurs organisés hebdomadairement dans différents lieux de Nairobi, dans les locaux de restaurants, voire pour l'un d'eux dans l'enceinte d'une ambassade. En 2007, une épicerie spécialisée a ouvert son approvisionnement aux producteurs locaux, suivie quelques années plus tard par une chaîne de supermarché. Approvisionnés par une centaine de marâ̂chers, ces débouchés permettaient en 2013 de vendre avec des marges sur les prix pouvant aller de 15 à plus de $194 \%$ (Tankam, 2015). Ces producteurs ne représentent qu'une petite fraction des milliers de producteurs formés à l'agriculture biologique mais n'ayant pas franchi l'étape de la certification (Taylor, 2006).

Si la production biologique certifiée reste limitée, la consommation n'en est, elle aussi, qu'à ses débuts. En 2012, un Kenyan sur deux indiquait ne pas connaître le terme «alimentation biologique», contre $13 \%$ en Ouganda, seuls $24 \%$ déclaraient connaître l'existence de certifications locales (Ndungu, 2013). Cette offre ayant essentiellement été poussée par des ONG pour sécuriser et améliorer les revenus des petits producteurs, elle a presque précédé la demande qui se révèle peu informée.

Face à l'absence de législation nationale et aux faibles revenus des producteurs, les premiers marchés biologiques locaux se sont appuyés sur un Système de contrôle interne (SCI), une forme de certification collective, proposé par Encert certificateur de droit privé kenyan (Taylor, 2006). En parallèle, les ONG locales ont poursuivi un travail de plaidoyer institutionnel au niveau national et sous-régional qui a débouché en 2007 sur la mise en place d'un label biologique est-africain nommé Kilimohai (EAC, 2007). Ce label reconnaît comme biologiques des produits soumis à une certification de premier ordre (autocertification), de second ordre (contrôle établi par l'acheteur) et par tiers ainsi que la possibilité de mettre en place des systèmes participatifs de garantie. Saisissant cette évolution institutionnelle, les producteurs en SCI l'ont abandonné, privilégiant un SPG pourtant désigné par la littérature économique classique comme moins crédible qu'une certification par tiers. Ce constat fait, nous interrogeons la capacité des marchés biologiques locaux kenyans à se maintenir dans le temps.

L'asymétrie d'information sur la qualité du produit (e.g. Akerlof 1970; Arrow, 1971) peut, pour partie, expliquer ce constat. La question est d'autant plus importante que les produits biologiques sont des biens de croyance (Nelson, 1970 ; Darby et Karni, 1973). Il s'agit de biens soumis à une asymétrie d'information particulière qui ne peut être révélée par l'aspect du bien ni par la consommation (Raynaud et Sauvée, 2000). Une majorité de travaux relevant de l'économie de l'information s'accorde à désigner comme biens de croyance des biens soumis à une certification par un tiers indépendant, luimême soumis à un mécanisme d'accréditation (Caswell et Mojduszka, 1996; Feddersen et Gilligan, 2001; Jahn et al., 
2005). Toutefois, un autre pan de l'économie s'est inscrit en faux tant sur les hypothèses comportementales des agents que sur le diagnostic de l'asymétrie informationnelle. Hirschman (1974) décrit des situations dans lesquelles ni les producteurs ni les consommateurs ne savent effectivement ce qui fait la qualité d'un produit, notamment dans le cas de marchés émergents. Dans ce cas, il s'agit d'une période durant laquelle les consommateurs n'ont pas précisément identifié leurs préférences et peuvent se trouver déçus par les biens et services proposés. La pérennité des marchés repose alors sur la recherche de voies d'expression et de rencontres entre consommateurs, producteurs et experts.

Dans les pays en développement, les filières de produits de qualité s'organisent dans des environnements institutionnels fragiles et peu outillés pour réguler ces marchés. Principalement considérées comme des moyens de lutte contre la pauvreté des petits producteurs, ces filières n'émergent pas en réponse à une demande qui par ailleurs est peu informée des caractéristiques de ces nouveaux produits (IFOAM, 2013). Elles sont initiées par des ONG locales qui se chargent de sensibiliser les premiers consommateurs (Goldberger, 2008). Par conséquent, asymétrie informationnelle et incertitude peuvent être alternativement ou simultanément des composantes de ces marchés. Aussi, nous posons la question de la capacité des marchés biologiques locaux à se maintenir dans le temps, c'est-à-dire à fournir aux consommateurs des informations crédibles et donc à réduire les risques de fraudes, tout en donnant la possibilité aux différentes parties d'exprimer leurs attentes quant à la qualité des produits. Nous nous appuyons sur le cas des marchés kenyans et nous complétons notre cadre d'analyse basé sur l'économie de l'information par des travaux ayant développé la question de l'incertitude partagée sur la qualité des biens (Hirschman, 1970, 1974, 1982 ; Lupton, 2005, 2015).
Dans une première partie, nous caractérisons les enjeux des marchés biologiques et interrogeons les spécificités des marchés biologiques locaux kenyans. Puis, nous présentons notre grille d'analyse des trois dispositifs de certification et de contrôle. Nous revenons sur notre méthodologie et enfin nous présentons nos résultats qui consistent à désigner le mode de contrôle le plus à même de prendre en charge ce double enjeu.

\section{Les systèmes de certification en économie et dans les pays en développement}

\section{Les conditions de pérennité des marchés de produits de qualité}

L'économie de l'information a permis de classer les biens de consommation en trois grandes catégories en fonction du coût d'accès à l'information sur leurs caractéristiques, les biens de recherche et d'expérience (Nelson, 1970) et les biens de croyance (Darby et Karni, 1973). Les biens de recherche sont des biens dont les caractéristiques sont connues avant l'achat, une tomate choisie pour sa couleur rouge, par exemple. Ici la recherche suppose l'inspection du bien avant achat. Dans le cas des biens d'expérience, il est moins coûteux pour le consommateur de connaître les caractéristiques en consommant le bien qu'en passant par une recherche. C'est le cas lorsque ce qui est recherché est le goût spécifique d'un produit. Pour ce qui est des biens de croyance, la seule consommation ne suffit pas et l'acquisition de l'information sur les caractéristiques du bien est prohibitive parce que trop coûteuse pour le consommateur non expert. Les produits biologiques appartiennent à cette catégorie de biens pour lesquels les marchés sont donc imparfaits (Coestier et Marette, 2004). C'est un premier niveau d'incertitude. Darby et Karni (1973) précisent aussi que ce sont des biens pour lesquels les consommateurs ne sont pas 
certains qu'ils satisfassent effectivement leurs besoins : "the consumer is unaware of the ability of the repair service to satisfy a given want $»^{1}$. La question n'est plus alors uniquement de savoir si ces biens ont les caractéristiques annoncées mais si ces caractéristiques vont répondre aux besoins des consommateurs. C'est donc un second niveau d'incertitude qualifiée d'incertitude de prescription (Dufeu, 2015). Ces deux niveaux sont souvent réduits en un seul dans la littérature, l'asymétrie d'information entre un producteur qui sait et un consommateur qui ne sait pas (Balineau et Dufeu, 2010). Considérons dans un premier temps l'asymétrie d'information au sens de l'économie de l'information.

L'intensité des problèmes contractuels dépend du type de produits et des coûts de mesure associés. Les difficultés d'accès à l'information sont renforcées dans le cas des biens de croyance dont la qualité n'est pas directement observable par des consommateurs non experts (Baummann, 2001; Giannakas, 2002; Jahn et al., 2005). Les produits biologiques sont définis par leur respect du cahier des charges et donc d'encadrement des étapes de production mais aussi de transformation. Le risque de fraude de la part du producteur est particulièrement élevé car l'accès à l'information sur le respect de l'ensemble de la chaîne est très coûteux pour le consommateur seul. Le consommateur est donc soumis à une asymétrie d'information particulièrement forte qui peut tout aussi bien être une situation de sélection adverse et d'aléa moral. La première qualifie une situation dans laquelle le consommateur manque d'information sur les caractéristiques d'un bien supposément connues par le producteur. Il y a risque d'aléa moral dès lors que le producteur peut choisir le niveau de qualité de son bien, y compris

1. «Le consommateur ignore la capacité d'un service de réparation à satisfaire un besoin donné. » (traduction des auteurs). après contractualisation. Ce problème est accentué quand le signal est une propriété collective et que certains producteurs peuvent fournir une qualité moindre tout en bénéficiant des investissements réalisés par le groupe (Raynaud et Sauvée, 2000 ; Winfree et McCluskey, 2005). Lorsque l'information redevient accessible aux consommateurs, les produits biologiques comme les produits d'expérience peuvent être rapprochés des biens de recherche (Caswell et Mojduszka, 1995 ; Achilleas et Anastatios, 2008).

La discussion porte alors sur les mécanismes favorisant la crédibilité du signal pour les consommateurs et donc la découverte de la fraude. La répétition des achats est insuffisante et la réputation ne protège le consommateur que dans le cas d'une probabilité élevée de détection de fraude (McCluskey, 2000 ; Engel, 2006). Cette probabilité augmente avec la fréquence des contrôles et le fait que ces derniers soient conduits par des agents extérieurs aux transactions, privés ou publics selon le niveau de qualité fixé et le coût associé (Roe et Sheldon, 2007), ainsi que l'existence de mécanismes de divulgation de l'information et de mauvaise publicité (Caswell et Mojduszka, 1996 ; McCluskey, 2000 ; Achilleas et Anastasios, 2008). Si les certifications sont censées offrir aux consommateurs des informations et des garanties, les signes ne sont pas équivalents dans la phase de qualification (nombre de critères retenus par exemple) comme dans celle de vérification. En effet, les modalités de contrôle résultent d'un compromis entre la précision de la mesure et les coûts nécessairement liés à cette mesure (Bougherara et Grolleau, 2004). Enfin, de nombreux travaux ont également souligné la concurrence entre certificateurs et ses effets sur l'indépendance et la sévérité des contrôles (Lizzeri, 1999 ; Anders et al., 2007 ; Fischer et Lyon, 2014). Le risque de fraude existe donc aussi sur les signaux eux-mêmes. 
Reconnaître que les consommateurs doivent être au centre de la définition de la qualité d'un produit, c'est faire l'hypothèse qu'ils connaissent leurs préférences et savent associer la satisfaction de cellesci aux attributs d'un produit (Achilleas et Anastasios, 2008). C'est une hypothèse rejetée par Hirschman (1970, 1974). Le concept d'ignorance partagée entre producteurs et consommateurs peut être rapproché du second type d'incertitude décrit par Darby et Karni (1973). Hirschman (1974) montre ainsi que, pour un certain nombre de biens et de services, le problème n'est pas une différence d'information mais une incapacité pour le producteur à répondre à la demande parce qu'il propose un produit ou un service sans connaître tous les critères effectivement à même de satisfaire la demande. De ce fait, le producteur propose une offre sans en connaître toutes les caractéristiques et sans que les consommateurs sachent ce qu'ils sont en mesure de demander. Ce cas de figure s'observe notamment lorsque la demande précède l'offre, celle-ci devant rapidement s'adapter à des consommateurs qui peuvent être en réalité peu précis sur les caractéristiques attendues. C'est le cas dans les phases d'émergence de marchés et au-delà puisque la qualification d'un produit n'est pas toujours fixée une fois pour toutes. Les produits complexes se trouvent ainsi soumis à ce que l'auteur nomme des « cycles d'acceptation » (Hirschman, 1974). La détérioration de la qualité d'un produit peut donner lieu à trois réactions du consommateur : la défection, la prise de parole ou la loyauté envers l'organisation fautive (Hirschman, 1970). La possibilité d'exprimer attentes et déceptions est alors un moyen d'éviter une défection définitive. Le refus du postulat du producteur omniscient est repris par Lupton (2005) qui propose une nouvelle catégorie de produits, les biens dits «indéterminés ». Il s'agit de biens incomplets qui ne sont pas définis de manière arrêtée ou unanime et pour lesquels il existe une incertitude partagée et radicale sur la qualité. Au cas de figure de l'émergence d'un nouveau produit, source d'incomplétude partagée avec Hirschman et Lupton (2005), se rajoute l'incertitude liée au passé ou aux impacts futurs de ce produit et utilisée de manière plus ou moins stratégique pour déstabiliser le marché. Considérant le cas de l'émergence d'un produit, le problème économique n'est plus uniquement celui de la fraude intentionnelle, mais aussi d'une possibilité d' «erreur de jugement» de la part du producteur, du fait de son ignorance conscientisée ou non de ce qui fait qualité, ou de son inexpérience. Dans ce cas, le risque d'effondrement du marché dû à une qualité inférieure aux attentes des consommateurs peut être minimisé par ce que Lupton (2009) appelle « l'option de la voix » en référence directe à Hirschman (1974).

\section{Les spécificités des marchés des produits biologiques en Afrique}

L'enjeu du contrôle s'avère peut-être plus prégnant en Afrique subsaharienne qu'ailleurs. En effet, bien que l'aléa moral soit un véritable problème en matière de certification environnementale, il peut s'en trouve atténué dans les pays développés dans la mesure où la conversion s'accompagne d'investissements initiaux importants (connaissances, compétences, matériel, temps de conversion) et pour certains irrécupérables, dont la perte en cas de découverte de tricherie serait trop coûteuse (Grolleau et Caswell, 2006). Par ailleurs, une fois acquises, ces capacités peuvent engendrer ce que ces auteurs désignent comme un «verrouillage automatique », dû à une stratégie triplement gagnante pour l'entreprise, le consommateur et l'environnement. Le risque d'aléa moral demeure au Kenya où la conversion n'implique pas d'investissements spécifiques. Ainsi, les enquêtes montrent que l'essentiel des dépenses va au recrutement de 
main-d'œuvre supplémentaire et non dans des actifs dédiés, puisque les nouvelles connaissances acquises en formation, par exemple, sont tout à fait transférables vers une activité non certifiée (Tankam, 2015). Par ailleurs, la profitabilité de ces produits reste posée dès lors que la vente avec premiums ne masque pas l'existence de coûts de transaction, des besoins en maind'œuvre renforcés, des coûts d'opportunité et une offre variant avec les saisons, fortement dépendante de la ressource en eau (Tankam, 2015). Il n'est donc pas possible de faire l'hypothèse de ce même verrouillage automatique. L'aléa moral n'est par ailleurs pas accentué par la dimension collective des SCI et des SPG, nos travaux ayant montré que le risque de capture des investissements réalisés par d'autres est en grande partie contenu par un contrôle de l'accès aux marchés de producteurs et de l'origine des produits vendus dans les épiceries spécialisées (Tankam, 2017). L'asymétrie informationnelle n'en reste pas moins un enjeu majeur sur ces marchés.

Toutefois, l'asymétrie d'information n'épuise pas tous les problèmes liés à l'échange de biens de qualité et il apparaît réducteur de résumer la satisfaction des besoins des consommateurs au seul respect de la certification par le producteur. En effet, les critères de satisfaction de la demande restent pour partie encore inconnus dans la mesure où les marchés de qualité sont davantage pensés comme des outils de lutte contre la pauvreté avec pour conséquence d'être en quelque sorte situés en amont à des besoins effectivement exprimés (UNEP, 2010). Les institutions internationales et les sociétés civiles locales font le pari d'une demande locale croissante pour les produits biologiques, tirée par des consommateurs des centres urbains appartenant à une classe moyenne supérieure et aisée, informés, et potentiellement de plus en plus soucieux du caractère sain de leur alimentation (Probst et al.,
2012 ; De Bon et al., 2017). Au Kenya, la réponse sous la forme d'une certification de fruits et légumes biologiques constitue une réponse parmi d'autres enjeux d'une alimentation saine et de qualité. D'autres pays auront fait des choix différents, le Vietnam, par exemple, avec une offre de légumes dits «propres » correspondant à une agriculture raisonnée et non biologique (Figuié et Mayer, 2010). De plus, l'offre kenyane est basée sur un référentiel européen, choix motivé par une recherche d'économie de coûts de transaction dans la définition de la norme et par la volonté de rassurer des consommateurs en se référant à des standards pouvant faire autorité (Tankam, 2017). Considérer le seul enjeu de l'asymétrie d'information reviendrait à dire que ce cahier des charges puisse spontanément satisfaire des consommateurs confrontés à d'autres réalités économiques et sociales. Or nous pouvons faire l'hypothèse que la démarche d'achat dans les pays en développement soit vécue par les consommateurs comme un soutien à l'agriculture familiale, encouragés d'ailleurs sur cette voie par les ONG locales physiquement présentes sur les marchés. C'est pourtant une dimension sociale absente du cahier des charges biologiques dans les pays développés. De même, nous pourrions faire l'hypothèse raisonnable d'un décalage entre les représentations que les consommateurs intéressés par une certification peuvent se faire des autres attributs des produits, en matière de packaging ou d'achalandage par exemple, et la distribution effective de ces produits. Les fruits et légumes sont en effet commercialisés en vrac sans étiquetage spécifique et sur des lieux de vente éphémères avec des investissements rudimentaires dans l'organisation des étals. En revanche, l'existence d'une incertitude partagée relative aux impacts passés ou futurs du bien, déstabilisatrice pour le marché, défendue par Lupton (2005) est plus difficile à argumenter. Ainsi, quand bien même les données 
sont lacunaires sur les impacts environnementaux et économiques de la production biologique en zone tropicale, il ne nous est pas possible d'en déduire des effets potentiellement déstabilisants pour des consommateurs.

À l'aune de ces éléments, il est possible de comprendre que les marchés locaux s'organisent autour d'une offre en cours de définition sans que les acteurs du marché, producteurs, premiers consommateurs et ONG ne soient nécessairement conscients de cette incertitude. Aussi l'enjeu porte-t-il à la fois sur le risque de comportement opportuniste et d'erreur de la part du producteur. Ce risque d'erreur pouvant être résolu par l'existence d'espaces d'ajustements mutuels entre les deux parties.

\section{Une grille d'analyse adaptée aux marchés biologiques africains}

Suivant à la fois les spécificités des marchés biologiques kenyans et les caractéristiques économiques des biens de croyance, notre grille d'analyse, principalement basée sur l'analyse de l'asymétrie d'information est complétée par le concept d'incertitude partagée (figure 1). Nous considérons également deux critères relatifs à l'économie de l'attention (Davenport et Bech, 2000). Ainsi huit critères sont proposés :

(i) Précision et étendue de la mesure : les contrôles sont source de coûts de transaction liés aussi bien à la définition des critères du contrôle qu'au contrôle des pratiques en lui-même et au suivi administratif post-contrôle. Par suivi administratif, nous faisons essentiellement référence au rapport qui sanctionne toute inspection et qui dresse des conclusions, recommandations ou obligations faites au producteur. Par conséquent, plus l'éventail des pratiques à contrôler est large et plus le temps du contrôle en lui-même et du suivi administratif est important. L'existence de coûts de mesure peut inciter à fixer un niveau de qualité plus bas confirmant le fait que la précision de la mesure est bien une variable d'ajustement (McCluskey, 2000 ; Carriquiry et al., 2003). Or une préférence pour des critères de contrôle réduit peut être synonyme d'un niveau de qualité lui-même réduit. Non seulement le niveau d'exigence ainsi limité est susceptible de s'accompagner d'une offre d'une qualité moindre, mais des critères trop vagues ou non identifiables peuvent aussi conduire à un label difficile à interpréter, ce qui peut rendre sa distribution difficile. Nous considérons donc, au titre de ce critère, l'étendue des normes sur lesquelles les producteurs sont contrôlés.

Plusieurs critères relatifs aux modalités des contrôles augmentent la probabilité de découverte des fraudes.

(ii) Degré d'indépendance du contrôleur: le contrôleur ${ }^{2}$ est responsable des opérations de « contrôle » ou de « vérification » selon qu'il s'agisse d'une certification par un tiers ou d'autres dispositifs tels que les SPG. À défaut de tenir compte de l'expertise du contrôleur, nous considérons son degré d'indépendance vis-à-vis de la définition des normes et de la production. En effet, il est communément admis en économie de l'information que plus le contrôleur est indépendant de ces deux phases et plus le risque de collusion est diminué (Tirole, 1986 ; Tanner, 2000 ; Busch et al., 2005). Par ailleurs, plusieurs travaux ont souligné l'importance de l'accréditation du certificateur par des institutions spécialisées pour s'assurer de l'objectivité du processus de certification (Manning et Baines, 2004 ; Deaton, 2004). Ainsi, nous observerons ici à la fois l'indépendance vis-à-vis du producteur et l'existence de mécanismes d'accréditation.

2. Dans certains cas, le contrôleur est différent du certificateur. Il peut être employé par lui. Dans d'autres, certificateur et contrôleur correspondent à la même personne. 
(iii) Type de contrôle : in situ/documents: dans le cas de SCI le certificateur peut procéder à des contrôles aléatoires des exploitations. Lorsque la certification se base sur les registres d'opérations agricoles tenues par les producteurs, les certificateurs font face au double risque d'opportunisme de la part des producteurs et d'erreur. En économie de l'information, l'asymétrie est essentiellement comprise comme donnant lieu à des risques de tricherie ou de mensonge délibérés. Cependant, la notion d'incertitude partagée reconnaît la possibilité d'une méconnaissance des producteurs quant aux attentes des consommateurs (Lupton, 2005). La possibilité d'erreur est renforcée ici, la compréhension des normes pouvant être compliquée par les difficultés d'écriture et de lecture de documents en langue anglaise. (iv et v) Fréquence des contrôles et niveau de sanction: la multiplication des contrôles augmente les probabilités de découverte de fraude (Jahn et al., 2005). Toutefois, dans une logique d'économie des coûts, il existe des arbitrages entre fréquence et niveau de sanction. En effet, il peut être privilégié de « pénaliser rarement mais lourdement » (Bougherara, 2003), la menace d'une lourde sanction pouvant être dissuasive. Ainsi, un dispositif de certification crédible, c'est-à-dire suffisamment contraignant pour réduire les risques d'opportunisme, devra intégrer des contrôles fréquents mais aussi une certaine progressivité dans les pénalités permettant de refléter l'importance de la non-conformité. C'est ce caractère progressif que nous considérerons dans ce cinquième critère.

Figure 1. Synthèse de la grille d'analyse des conditions de pérennité des dispositifs de certification des produits biologiques au Kenya

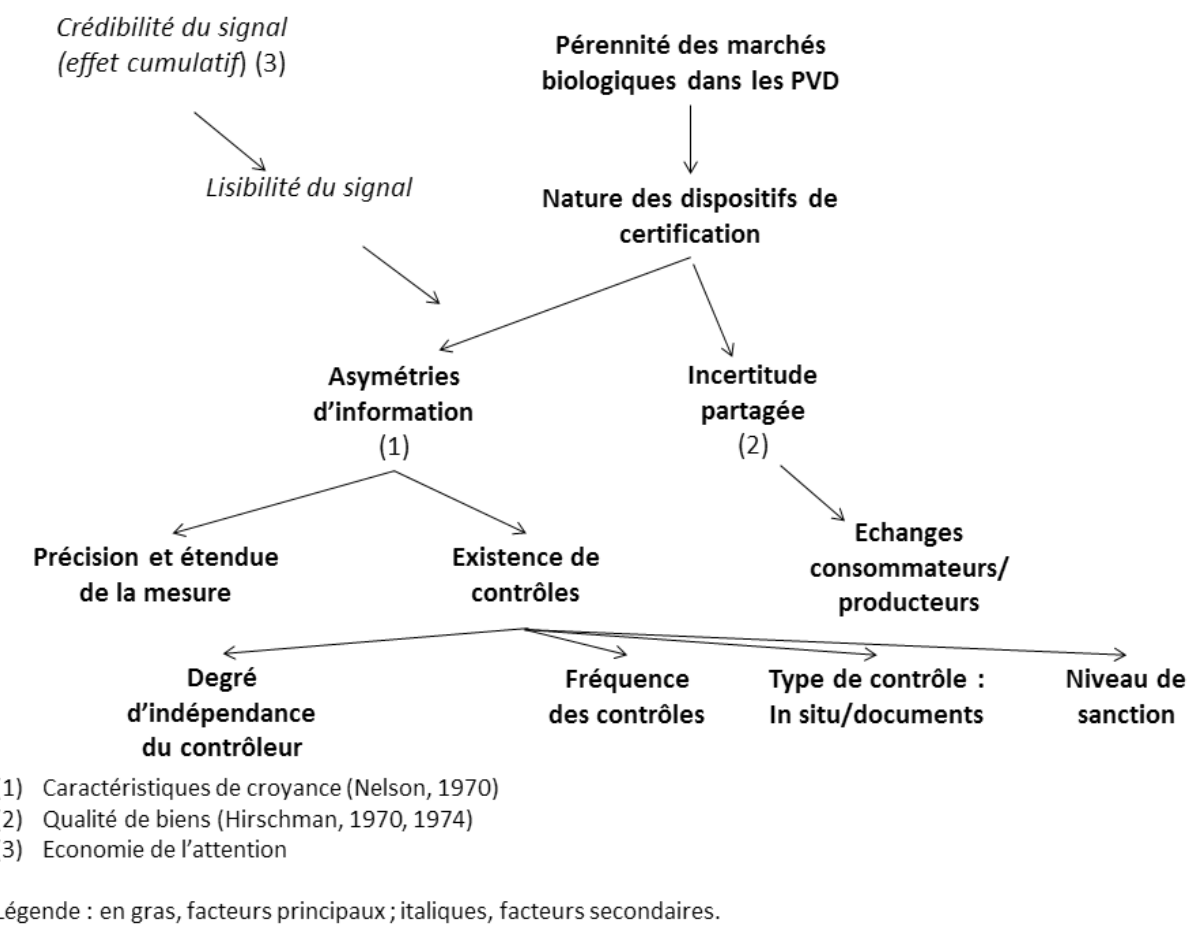

Source : les auteurs. 
RECHERCHE

(vi) Les phases d'émergence de nouveaux marchés nécessitent des ajustements successifs sur la qualité qui satisfera effectivement les attentes en cours de définition des consommateurs. L'enjeu n'est donc pas de protéger les consommateurs contre des comportements frauduleux mais de donner la possibilité à la co-construction de la qualité de se réaliser. Nous retenons ici comme critère la possibilité de rencontres entre consommateurs et producteurs permettant aux premiers de verbaliser leurs attentes et aux seconds d'ajuster le produit. (vii) Lisibilité $d u$ signal : pour les entreprises et les dispositifs de certification, il est au moins aussi important de fournir de l'information que de capter l'attention dans un contexte de surabondance d'information. Le problème majeur des consommateurs n'est pas forcément d'acquérir de l'information mais d'avoir à disposition des «repères » permettant de résumer les connaissances (Valceschini et Mazé, 2000 ; Bougherera et Grolleau, 2004). Comme le soulignent les travaux sur l'économie de l'attention (Davenport et Bech, 2000), l'asymétrie d'information est très dépendante des stratégies de communication, notamment des conditions d'utilisation et de visibilité des logos.

(viii) Crédibilité du signal: une fois le raccourci informationnel utilisé et capté, la marque peut être associée à des attributs positifs si celle-ci est affectée d'une crédibilité importante. Elle représente un effet cumulatif de l'ensemble des actions marketing engagées précédemment par l'entreprise (Erdem et al., 2002).

\section{Méthodologie}

\section{Le cas d'étude}

Notre étude nous amène à considérer deux grands modes de certification et de contrôle

Tableau 1. Les trois principaux dispositifs de certification et de contrôle des produits biologiques kenyans

\begin{tabular}{|c|c|c|c|}
\hline & \multicolumn{2}{|c|}{ Certification par tiers } & $\begin{array}{l}\text { Dispositif de contrôle } \\
\text { Système participatif de garantie (SPG-N) }\end{array}$ \\
\hline $\begin{array}{l}\text { Nature du } \\
\text { dispositif }\end{array}$ & \multicolumn{2}{|c|}{ Certification par tierce partie via l'organisme certificateur privé Encert. } & $\begin{array}{l}\text { Systèmes d'assurance qualité orientés } \\
\text { localement. Certification sur la base d'une } \\
\text { participation active des acteurs concernés. }\end{array}$ \\
\hline Date & Depuis 2005 & De 2005 à 2010 & Depuis 2013 \\
\hline Norme & \multicolumn{2}{|c|}{$\begin{array}{l}\text { Cahier des charges d'ENCERT puis évolution progressive vers la norme est- } \\
\text { africaine à partir de } 2007 \text {. }\end{array}$} & Norme est-africaine \\
\hline & \multicolumn{3}{|c|}{ Maraîchage, arbres fruitiers (maïs, haricots, choux, mangues, bananes) et petit élevage. } \\
\hline \multirow[b]{2}{*}{$\begin{array}{l}\text { Mode de } \\
\text { commercialisation }\end{array}$} & \multicolumn{3}{|c|}{ Une partie peut être vendue sur des marchés conventionnels sans premium. } \\
\hline & $\begin{array}{l}\text { Sur marchés de producteurs } \\
\text { ou épiceries/supermarchés, } \\
\text { possibilité de déléguer la } \\
\text { vente. }\end{array}$ & $\begin{array}{l}\text { Sur marchés de producteurs } \\
\text { principalement, en personne et en } \\
\text { présence des membres des } \\
\text { groupements. }\end{array}$ & $\begin{array}{l}\text { Sur marchés de producteurs ou } \\
\text { épiceries/supermarchés, possibilité de déléguer } \\
\text { la vente. }\end{array}$ \\
\hline Raisons du choix & $\begin{array}{l}\text { Accéder aux marchés } \\
\text { biologiques locaux; Refus } \\
\text { d'intégrer une démarche } \\
\text { collective, moyens } \\
\text { économiques suffisants. }\end{array}$ & $\begin{array}{l}\text { Accéder aux marchés biologiques locaux } \\
\text { mais moyens économiques limités. } \\
\text { Le } \mathrm{SCl} \text { permet en moyenne de diviser les } \\
\text { coûts par } 16 \text { par rapport à la certification } \\
\text { par tiers individuelle (soit un total de } 25 \\
\text { euros). }\end{array}$ & $\begin{array}{l}\text { Accéder aux marchés biologiques locaux mais } \\
\text { moyens économiques limités. } \\
\text { Volonté de récupérer le choix des modalités de } \\
\text { commercialisation, refus des coûts de } \\
\text { transaction associés à la vente directe en } \mathrm{SCl} \text {. } \\
\text { Par rapport à la certification par tiers } \\
\text { individuelle, coût direct divisé par } 40 \text { (soit un } \\
\text { total de } 10 \text { euros), correspondant aux seuls frais } \\
\text { d'adhésion à l'association. }\end{array}$ \\
\hline
\end{tabular}


que nous déclinons entre trois modalités, le SCI étant bien une forme possible de certification par tiers (tableau 1). Certification individuelle et SCI préexistaient à la norme est-africaine promulguée en 2007. Cette dernière a donné le droit d'utiliser la dénomination «agriculture biologique » aux certifications de premier ordre, de second ordre, aux certifications locales existantes donc aux SCI et aux SPG. Elle a remplacé les cahiers des charges existant sur les items communs. Les domaines non pris en charge par le nouveau référentiel (par exemple l'aquaculture) sont restés contrôlés sur la base des cahiers des charges antérieurs. Toutefois, la migration a été facilitée par l'ancrage commun du cahier des charges d'Encert et du label estafricain dans les principes biologiques de l'IFOAM et du codex alimentarius. Les producteurs se sont saisis de cette évolution institutionnelle pour remplacer progressivement les SCI par des SPG. Les acteurs nommés ici sont les producteurs mais aussi les consommateurs, les commerçants ou encore les ONG.

\section{Une méthode de collecte basée sur des entretiens semi-directifs}

Après une enquête préliminaire ayant permis d'identifier l'organisation des différentes filières et l'environnement institutionnel du secteur biologique domestique, nous avons procédé à des entretiens semidirectifs durant en moyenne $1 \mathrm{~h} 30$ à $2 \mathrm{~h}$. L'enquête a eu lieu en octobre et novembre 2013. Nous avons ainsi ciblé un SPG que nous nommerons le SPG-N approvisionnant régulièrement les marchés biologiques de Nairobi, héritier de deux précédents SCI fusionnés. Au sein de ce SPG, nous avons conduit des entretiens auprès de huit producteurs dont sept investis dans trois comités (inspection, sanction et marketing) qui rythment la vie de l'organisation. Leur implication dans le SCI et leur participation à la mise en œuvre des règles du groupement constituaient des sources d'information sur le fonctionnement global de cette certification groupée et sur les modes de prise de décision. Par ailleurs, nous avons également réalisé un entretien avec le chef du groupement à l'initiative du SPG. Trois des cinq producteurs certifiés individuellement ont également été enquêtés. Le premier est propriétaire d'une épicerie biologique de Nairobi. Il vend ses propres produits cultivés sur six hectares et s'approvisionne en complément auprès de producteurs indépendants. La seconde exploitation d'une superficie de deux hectares appartient à un ancien membre d'une ONG de développement local qui commercialise principalement sur des marchés de producteurs et auprès de restaurants spécialisés. La troisième est une entreprise de conseil aux agriculteurs, propriétaire de sa propre exploitation de deux hectares et qui écoule également ses produits sur les marchés biologiques domestiques. En dehors des groupements de producteurs, nous avons réalisé des entretiens avec l'organisme certificateur privé, des acteurs institutionnels (services centraux du ministère de l'Agriculture et chargés de service au bureau national des standards) et des membres des structures d'appui aux producteurs (ONG impliquées dans l'accompagnement des producteurs). Les entretiens ont été l'occasion de collecter divers documents stratégiques : le règlement intérieur du SPG, le cahier des charges dont les producteurs se sont dotés ou encore les documents d'inspections utilisés lors du contrôle des agriculteurs. Enfin, l'observation participante sur les marchés a porté plus spécifiquement sur les relations entre producteurs et consommateurs.

\section{Comparaison des dispositifs de certification}

Notre principal matériau est la caractérisation des différents dispositifs à partir des huit critères présentés plus haut. Les cinq premiers font référence à l'économie de l'information. Les travaux d'Hirschman et l'économie de l'attention contribuent 
respectivement à hauteur d'un et de deux critères. Les deux derniers critères de l'économie de l'attention sont moins centraux dans notre analyse qui porte principalement sur l'asymétrie d'information et l'incertitude partagée mais sont bien complémentaires des autres. Aussi sont-ils présentés ici comme des critères secondaires. Il convient de préciser que nous ne proposons pas non plus de pondération spécifique des six premiers critères. Toutefois, nous reconnaissons le fait qu'ils ne sont pas égaux. Nous le matérialisons en les faisant apparaître de manière distincte dans le tableau 3. Chaque critère est apprécié d'après trois niveaux (1,2 ou 3 étoiles). L'attribution des étoiles est réalisée en relatif en comparant les dispositifs entre eux. Nous ne faisons pas la somme de ces critères qui n'ont pas tous le même statut mais proposons une analyse qualitative de la performance de ces systèmes en nous appuyant sur une classification résumée en deux tableaux (tableaux 2 et 3 ).

\section{Des résultats qui montrent la portée des systèmes de certification et de contrôle}

Le SCI et la certification individuelle présentent des performances somme toute comparables, mais variables selon les critères de l'asymétrie d'information (tableaux 2 et 3). La certification individuelle se démarque par l'externalisation des contrôles réalisés annuellement sur l'ensemble des parcelles et sur le plan de la norme. Elle s'appuie, en effet, sur un cahier des charges relativement étendu de 110 règles organisant la production, le transport, la certification et la transformation. Le paragraphe dédié aux intrants précise pour 30 types de fertilisants différents les interdictions formelles et les pratiques admises. La certification participative s'appuie sur le même cahier des charges mais marque le pas sur la nature du contrôle in situ réalisé par un comité de producteurs désignés en interne. Ces inspections sont néanmoins plus fréquentes et correspondent bien à des temps d'inspection prévus ou inopinés :

"We have to inspect each field and every farmers to make sure they are doing what is in the guidelines ${ }^{3}$. (Membre du comité)

Un contrôle externe annuel est réalisé par Encert sur la base des registres individuels et des documents du groupement. En certification individuelle ou collective, Encert est lui-même soumis à un mécanisme d'accréditation par un indépendant. Les deux dispositifs ont en commun de reposer sur un système de sanction graduel et prévoient une notification écrite avec possibilité de deux rappels avant expulsion.

Concernant les modalités de contrôle, les différences entre le SPG et le SCI sont légères dans la mesure où il existe bien un double niveau de contrôle, interne par les producteurs, et externe par une des principales ONG locales (chargée de vérifier le respect du caractère participatif et démocratique du SPG) ainsi que par un comité de sanction des pratiques à majorité externe. En interne, les inspections sont réalisées par neuf producteurs et ne s'apparentent pas à du conseil comme on aurait pu l'attendre dans un SPG mais bien à des contrôles :

«Using a checklist, the team holds farm visits to check whether members are following the group internal standard ${ }^{4}$. » (Membre du comité)

Ce comité transmet les rapports à un comité chargé de sanctions composé de trois personnes, un représentant des producteurs, un représentant du ministère de

3. « Nous devons inspecter chaque champ et tous les agriculteurs pour nous assurer qu'ils respectent le cahier des charges. »

4. «À l'aide d'une liste de contrôle, l'équipe organise des visites pour vérifier si les membres respectent la certification interne. » 
l'Agriculture et un membre d'une ONG kenyane. Les contrôles sont cependant moins fréquents qu'en SCI.

En revanche, l'enjeu majeur réside davantage dans la transition non perçue par les consommateurs entre le SCI et le SPG, engendrant ainsi une asymétrie d'information particulièrement forte entre eux et les producteurs. En effet, le cahier des charges du SPG jusqu'alors utilisé a été significativement allégé avec un corpus réduit à dix règles choisies par les producteurs dont cinq portent sur les pratiques agricoles - principalement sur les intrants et sur les mesures de protection face aux risques de contamination (les mentions relatives aux haies par exemple). De plus, après délibération, les conditions d'admission dans le groupement ont été assouplies puisque les préconisations de la norme estafricaine d'une année minimum de conversion ont été supprimées. Il est uniquement indiqué qu'un producteur souhaitant adhérer au SPG est soumis à une période de probation de deux mois à l'issue de laquelle il est ou non admis sur la base de sa motivation et non de ses pratiques. Or ces différents assouplissements - notamment l'évolution de la norme - qui correspondent à une réappropriation et une phase d'apprentissage pour les producteurs de ce que peut recouvrir l'agriculture biologique localement, sont rendus peu visibles dans la mesure où les signes de qualité sont restés les mêmes. En témoignent les critères relevant de l'économie de l'attention qui ne permettent de distinguer aucun des systèmes. Dans les deux premiers dispositifs, les producteurs arborent le logo d'Encert, qu'il soit photocopié sur leurs produits ou sur des bannières. Les producteurs en SPG étant les mêmes qu'en SCI, ils bénéficient ainsi des investissements réalisés par Encert pour créer sa réputation (formation en Europe du certificateur, logo, site internet). En dehors d'une actualisation des

\section{Tableau 2. Organisation du contrôle sur les marchés domestiques kenyans}

\begin{tabular}{|c|c|c|c|c|}
\hline $\begin{array}{l}\text { Courant } \\
\text { théorique }\end{array}$ & Critères & $\begin{array}{l}\text { Certification par un tiers } \\
\text { individuelle }\end{array}$ & $\begin{array}{l}\text { Système de contrôle interne } \\
\qquad(\mathrm{SCl})\end{array}$ & $\begin{array}{c}\text { Système participatif de } \\
\text { garantie (SPG) }\end{array}$ \\
\hline \multirow{5}{*}{$\begin{array}{l}\text { Asymétrie } \\
\text { d'information }\end{array}$} & $\begin{array}{l}\text { 1. Précision et } \\
\text { étendue de la } \\
\text { mesure }\end{array}$ & $\begin{array}{l}\text { Cahier des charges Encert } \\
110 \text { règles relatives aux } \\
\text { pratiques agricoles } \\
\text { puis norme est-africaine } 38 \\
\text { règles }\end{array}$ & $\begin{array}{l}\text { Cahier des charges Encert } \\
110 \text { règles relatives aux pratiques } \\
\text { agricoles et à l'organisation du SCI }\end{array}$ & $\begin{array}{l}10 \text { règles dont } 5 \text { sur les pratiques } \\
\text { agricoles choisies en assemblée } \\
\text { générale par les producteurs à } \\
\text { partir du cahier des charges est- } \\
\text { africain }\end{array}$ \\
\hline & $\begin{array}{l}\text { 2. Degré } \\
\text { d'indépendance } \\
\text { du contrôleur }\end{array}$ & $\begin{array}{l}\text { Contrôle exercé par Encert } \\
\text { (accrédité par l'IOAS) }\end{array}$ & $\begin{array}{l}\text { Contrôle principalement interne et } \\
\text { des pratiques et contrôle externe } \\
\text { des documents } \\
\text { (accrédité par l'IOAS) }\end{array}$ & $\begin{array}{l}\text { Contrôle interne des pratiques et } \\
\text { contrôle externe des documents } \\
\text { (non accrédité) }\end{array}$ \\
\hline & $\begin{array}{l}\text { 3. Type de } \\
\text { contrôle: In } \\
\text { situ/Documents }\end{array}$ & In situ & $\begin{array}{l}\text { Documents réalisés par les } \\
\text { producteurs (pratiques et } \\
\text { fonctionnement du groupement) } \\
\text { et in situ sur quelques } \\
\text { exploitations }\end{array}$ & $\begin{array}{l}\begin{array}{l}\text { Documents réalisés par les } \\
\text { producteurs (pratiques et } \\
\text { fonctionnement du groupement) }\end{array}\end{array}$ \\
\hline & $\begin{array}{l}\text { 4. Fréquence des } \\
\text { contrôles }\end{array}$ & 1 contrôle par an & 1 contrôle mensuel & 2 contrôles par an \\
\hline & $\begin{array}{l}\text { 5. Niveau de } \\
\text { sanction }\end{array}$ & $\begin{array}{l}\text { Sanctions progressives } \\
\text { prévoyant exclusion en cas } \\
\text { de non recours auxintrants }\end{array}$ & $\begin{array}{l}\text { Sanctions progressives prévoyant } \\
\text { exclusion en cas de non recours } \\
\text { auxintrants }\end{array}$ & $\begin{array}{l}\text { Sanctions progressives prévoyant } \\
\text { exclusion en cas de non recours } \\
\text { auxintrants }\end{array}$ \\
\hline $\begin{array}{l}\text { Incertitude } \\
\text { partagée }\end{array}$ & $\begin{array}{l}\text { 1. Echanges } \\
\text { consommateurs/ } \\
\text { producteurs }\end{array}$ & $\begin{array}{l}\text { Vente directe } \\
\text { intermédiée }\end{array}$ & $\begin{array}{l}\text { Vente directe par le producteur } \\
\text { systématique sur les marchés de } \\
\text { producteurs }\end{array}$ & $\begin{array}{l}\text { Vente directe et intermédiée } \\
\text { Absence de consommateur dans le } \\
\text { fonctionnement du SPG }\end{array}$ \\
\hline \multirow{2}{*}{$\begin{array}{l}\text { Economie de } \\
\text { l'attention }\end{array}$} & $\begin{array}{l}\text { 1. Lisibilité du } \\
\text { signal }\end{array}$ & $\begin{array}{l}\text { Logo du certificateur Encert } \\
\text { affiché sur les lieux de vente }\end{array}$ & $\begin{array}{l}\text { Logo du certificateur Encert affiché } \\
\text { sur les lieux de vente } \\
\text { Marque du groupement (OFM) }\end{array}$ & $\begin{array}{l}\text { Logo du certificateur Encert affiché } \\
\text { sur les lieux de vente } \\
\text { Marque du groupement (OFM) }\end{array}$ \\
\hline & $\begin{array}{l}\text { 2. Crédibilité du } \\
\text { signal }\end{array}$ & Site web d’Encert actualisé & $\begin{array}{l}\text { Régularité de la présence sur les } \\
\text { marchés de producteurs } \\
\text { Site web d'Encert actualisé }\end{array}$ & $\begin{array}{l}\text { Régularité de la présence sur les } \\
\text { marchés de producteurs } \\
\text { Site web d'Encert actualisé }\end{array}$ \\
\hline
\end{tabular}

Source : les auteurs. 
RECHERCHE

noms des producteurs certifiés sur son site web, le certificateur reconnaît avoir renoncé à dénoncer cette usurpation étant donné l'environnement institutionnel kenyan peu protecteur :

«As long as it will not be mandatory, they will sell on local markets without certification. The national policy draft was two years ago, and now what? I prefer to concentrate on international certifications and inspections ${ }^{5}$.

Là où le caractère émergeant des marchés nécessitait déjà des espaces d'échanges entre producteurs et consommateurs, cette phase d'apprentissage collective et de requalification du produit (en l'occurrence dans le sens d'assouplissements), masquée par la superposition de signaux, conforte le besoin d'une « option de la voix » (Hirschman, 1974; Lupton, 2005).

Pourtant, le système privilégié par les producteurs est le SPG qui s'avère plus faible dans la prise en charge de l'incertitude partagée, contrairement au SCI abandonné et une nouvelle fois plus performant. Une différence majeure entre SCI et SPG est, en effet, l'obligation dans le premier pour les paysans de vendre sur des «Points de Vente Communs ». Au Kenya mais aussi en Inde ou au Brésil, les SCI «ne permettent pas la commercialisation individuelle des produits » (IFOAM, 2013 ; Khosla, 2006 ; Rundgren, 2007 ; Källander, 2008). La suppression des intermédiaires est imposée afin d'éliminer des sources d'aléas supplémentaires dans un système où le contrôle par le certificateur est déjà indirect. Ce mode de commercialisation est organisé par le bureau du SCI :

«We coordinate, bringing farmers together, making sure that all of them will come here. It is compulsary to come here each Saturday, if they cannot, they have to call $u s^{6}$. » (Président d'un groupement de producteurs)

Tableau 3. Comparaison des dispositifs de certification et de vérification

\begin{tabular}{|c|c|c|c|c|}
\hline $\begin{array}{l}\text { Courant } \\
\text { théorique }\end{array}$ & Critères & $\begin{array}{l}\text { Certification par un } \\
\text { tiers individuelle }\end{array}$ & $\begin{array}{c}\text { Système de } \\
\text { contrôle interne } \\
\text { (SCI) }\end{array}$ & $\begin{array}{c}\text { Système } \\
\text { participatif de } \\
\text { garantie (SPG) }\end{array}$ \\
\hline \multirow{5}{*}{$\begin{array}{l}\text { Asymétrie } \\
\text { d'information }\end{array}$} & $\begin{array}{l}\text { 1. Précision et étendue de } \\
\text { la mesure }\end{array}$ & $* * *$ & $* * *$ & $* *$ \\
\hline & $\begin{array}{l}\text { 2. Degré d'indépendance } \\
\text { du contrôleur }\end{array}$ & $* * *$ & $* *$ & ** \\
\hline & $\begin{array}{l}\text { 3. Type de contrôle: In } \\
\text { situ/Documents }\end{array}$ & $* *$ & $*$ & $*$ \\
\hline & 4. Fréquence des contrôles & $*$ & $* * *$ & ** \\
\hline & 5. Niveau de sanction & $* *$ & $* *$ & $* *$ \\
\hline $\begin{array}{l}\text { Incertitude } \\
\text { partagée }\end{array}$ & $\begin{array}{l}\text { 1. Echanges } \\
\text { consommateurs/ } \\
\text { producteurs }\end{array}$ & $*$ & $* * *$ & $* *$ \\
\hline \multirow{2}{*}{$\begin{array}{l}\text { Economie de } \\
\text { l'attention }\end{array}$} & 1. Lisibilité du signal & $* *$ & $* *$ & $* *$ \\
\hline & 2. Crédibilité du signal & $* *$ & ** & $* *$ \\
\hline
\end{tabular}

5. « Tant que ce ne sera pas obligatoire, ils vendront sur les marchés locaux sans certification. Le projet de loi c'était il y a deux ans, et depuis quoi ? Je préfère me concentrer sur les certifications et les inspections au niveau international. »
6. « Nous nous chargeons de la coordination des agriculteurs en les réunissant et en veillant à ce qu'ils viennent tous ici. Il est obligatoire de venir ici chaque samedi. S'ils ne le peuvent pas, ils doivent nous appeler. » 
Cette obligation est rendue possible par la cession partielle des droits de propriété sur la production au bureau du groupement acceptée par chaque producteur (Tankam, 2017). Comme l'a montré l'observation, la vente directe peut donner la possibilité de discussions avec les consommateurs allant au-delà de la négociation des prix. Les producteurs répondent à des questions sur leurs pratiques, le fonctionnement de la certification, l'organisation des collectifs auxquels ils appartiennent, etc. Ces transactions répétées permettent donc au consommateur d'interroger la prise en compte de ses attentes et de donner à d'autres dimensions la possibilité d'émerger, et aux producteurs d'avoir accès à ces réflexions pour pouvoir ensuite faire évoluer l'offre. L'obligation de vente directe ouvre donc fortement la possibilité d'ajustements mutuels en SCI. À l'exception du magasin, lui-même producteur, qui peut échanger avec les clients venus acheter des fruits et légumes, les agriculteurs en certifications par tiers commercialisent principalement via des intermédiaires.

Le passage du SCI au SPG s'explique en partie par la volonté pour les producteurs de récupérer le choix de commercialisation de leurs produits, à la fois pour réduire les coûts d'opportunités liés à la vente directe groupée et pour diversifier les débouchés (Tankam, 2017). Toutefois, parce qu'ils sont parvenus à fidéliser des consommateurs, les producteurs continuent en partie d'approvisionner ces marchés urbains, en personne ou en déléguant les ventes. Ils sont donc ici plus performants sur l'incertitude partagée que la certification privée mais moins que le SCI qui n'autorisait pas cette délégation. Par ailleurs, aucun consommateur ni même transformateur ne s'est effectivement investi dans ce SPG, comme le reconnaît un des membres :

"There is a plan to involve buyers in the peer reviews in the future. The main challenge with regards to the involvement of stakeholders is their lack of time/interest: buyers and consumers usually say that they do not have time for the peer review activities or group meetings ${ }^{7}$. »

Ainsi, une étude de l'IFOAM notait à propos du SPG étudié ici en comparaison avec le SCI que «la différence entre ces systèmes [...] est effectivement très faible $[\ldots] »$ et concluait que « de ce point de vue, l'étiquette "participative" pourrait être un peu trompeuse » (IFOAM, 2013). Ceci explique également une moindre prise en charge de l'incertitude partagée par le SPG, là où le caractère émergent des marchés et la transition d'avec le SCI renforçaient pourtant le besoin d'échanges entre producteurs et consommateurs.

\section{Limites et prolongements de l'analyse}

La grille proposée a mis en lumière des proximités et des différences entre les trois dispositifs de contrôle de la production biologique vendue localement au Kenya. Il serait utile de l'utiliser dans d'autres contextes économiques et institutionnels où certification par tiers et alternatives se côtoient, voire de la mobiliser pour d'autres dispositifs, y compris au sein des SPG dont les modalités diffèrent selon les pays et les initiatives. En effet, bien que la vente directe obligatoire ne soit pas une spécificité des SCI kenyans, cette caractéristique oriente nos conclusions sur le dispositif et une comparaison avec des SCI différents pourrait aboutir à d'autres résultats.

La seconde limite est liée à la méthodologie d'évaluation de chaque critère qui, si elle s'appuie sur un matériau empirique

7. «Il est prévu d'impliquer les acheteurs dans les contrôles entre pairs à l'avenir. Le principal défi en ce qui concerne l'implication des parties prenantes est leur manque de temps/d'intérêt : les acheteurs et les consommateurs disent généralement qu'ils n'ont pas le temps pour les activités de contrôle entre pairs ou les réunions de groupe. » 
le plus enrichi possible, passe nécessairement par le prisme de notre compréhension des mécanismes et donc par des biais cognitifs. De plus, nous avons assumé de mettre sur un même plan les six premiers critères, considérant par exemple avec le même poids l'indépendance du contrôle et leur fréquence. Ce choix peut être questionné. En effet, nous estimons que les contrôles mensuels en SCI méritent de distinguer ce dispositif, comparativement aux deux autres et lui attribuons pour cela un nombre maximal d'étoiles. Cette analyse ne permet pas de tenir compte du fait que les contrôles, certes bien plus fréquents en certification groupée, n'en sont pas moins mis en œuvre par des producteurs, soit avec une indépendance quasi nulle. Il est possible d'envisager que l'indépendance du contrôle mérite une pondération qui reflète en quelque sorte l'importance de ce critère dans la littérature. Nous sommes conscients de cette limite mais à moins que cette supériorité sur les cinq autres critères ne soit effectivement démontrée, ce qui n'est pas fait à notre connaissance, nous assumons la grille d'analyse ainsi formulée. En revanche, nous proposons comme piste d'approfondissement du fonctionnement des SPG un cadre analytique allant au-delà du seul critère des échanges entre producteurs et consommateurs. Nous pourrions ainsi revenir davantage sur la dimension d'apprentissage revendiquée par les SPG, ou encore le fait qu'ils permettent plus facilement aux cahiers des charges de s'adapter aux caractéristiques locales, qui n'est pas analysé en tant que tel ici.

Par ailleurs, notre proposition de comparaison des dispositifs pourrait être prolongée par des données sur les coûts de ces derniers. Le SPG conçoit des coûts de transaction supplémentaires pour les agriculteurs associés à la définition au choix des normes et à la mise en œuvre des inspections, ainsi que des coûts d'opportunité. Concernant le SPG de Nature et
Progrès Tarn, le rapport final Pilot Project, Group certification in Europe mentionne par exemple un important travail bénévole (Van den Akker, 2009). De même, il serait judicieux d'intégrer les coûts de formation que les producteurs doivent supporter. En effet, dans le cas des SPG comme des SCI, les producteurs impliqués dans les bureaux des associations ou dans les activités d'inspections sont systématiquement formés à ces différentes tâches. Enfin, les producteurs du SPG évoquent également des demandes de défraiement des coûts de transports de la part des représentants du ministère et de l'ONG participant au comité de sanction.

$$
\text { * }
$$

Les caractéristiques des produits biologiques et du secteur agricole kenyan montrent qu'il existe bien un double enjeu d'asymétrie d'information et d'incertitude partagée pour le maintien des marchés et que ce double enjeu est différemment pris en compte par les dispositifs étudiés.

Lorsque l'on élargit l'analyse de l'asymétrie d'information à la prise en compte de l'incertitude partagée, les dispositifs qui ressortent comme étant les plus crédibles ne sont pas les certifications individuelles par un tiers. Si l'on considère notamment la possibilité d'ajustements mutuels entre producteurs et consommateurs mais aussi la fréquence des contrôles, les certifications groupées présentent une efficacité légèrement supérieure. S'il est possible de discuter de l'efficacité de contrôles réguliers mais opérés par les agriculteurs euxmêmes, cela met néanmoins en lumière les atouts du SCI et de son obligation, souvent considérée sous un angle négatif car perçue comme trop contraignante, de vente directe. En effet, cette obligation constitue un espace d'expression important pour les consommateurs dans cette phase d'émergence de nouveaux produits et marchés et d'apprentissage pour les producteurs. 
Si les différences sont réduites entre SCI et certifications par un tiers, elles sont en revanche plus marquées avec les SPG. Ainsi, l'analyse montre que les producteurs en SPG non seulement réalisent des contrôles sur la base d'un nombre très restreint de pratiques agricoles, mais que le contrôle direct est essentiellement réalisé par les producteurs eux-mêmes. Or les signaux apposés sur les produits étant restés les mêmes, cette double évolution n'a pas nécessairement été rendue visible pour les consommateurs. Face à un risque donc renforcé de déception, les SPG présentant l'avantage de permettre une adaptation de la norme au contexte local s'avèrent pourtant une nouvelle fois moins performants dans la création d'espaces d'ajustements mutuels entre producteurs et consommateurs. Aussi, nous montrons que les SPG kenyans qui ont remplacé les SCI disposent d'une marge de progression importante.
La mise en place d'un label public en 2007 a permis de contenir l'asymétrie informationnelle. Cependant, le label n'est pas parvenu à résoudre l'incertitude sur les contours des produits biologiques destinés au marché local dont il a pourtant renforcé l'importance en donnant la possibilité aux producteurs de définir l'étendue de la norme à respecter. Notre conclusion pourrait de ce point de vue être pessimiste sur le maintien des marchés et sur l'opportunité de ce label. Mais il semblerait plus juste, au regard de l'historique de l'agriculture biologique (le cas français est à ce titre intéressant), de considérer que ce qui se passe actuellement sur ces marchés relève d'agencements marchands qui continuent d'interroger la recherche. Une des pistes étant, comme nous l'avons montré, de faire dialoguer plusieurs cadres théoriques pour mieux comprendre l'émergence des marchés biologiques dans les pays en développement. 


\section{RÉFÉRENCES BIBLIOGRAPHIQUES}

Anders S., Monteiro D. M. S., Rouviere E. (2007). Objectiveness in the Market for Third-Party Certification: Does market structure matter? International Marketing and International Trade of Quality Food Products, $\mathrm{n}^{\circ}$ 651, pp. 651-663.

Akerlof G. (1970). The market for "lemons": Quality uncertainty and the market mechanism. The Quarterly Journal of Economics, vol. 84, n 3 , pp. 488-500.

Achilleas K., Anastasios S. (2008). Marketing aspects of quality assurance systems: The organic food sector case. British Food Journal, vol. 110, n 8, pp. 829-839.

Arrow K. J. (1971). The value of and demand for information. Decision and Organization, $\mathrm{n}^{\circ} 2$, pp. 131-139.

Balineau G., Dufeu I. (2010). Are Fair Trade goods credence goods? A new proposal, with French illustrations. Journal of Business Ethics, vol. 92, n 2, pp. 331-345.

Baummann P. (2001). Securing against fraud. In: Proceedings of the European Conference Organic Food and Farming. Copenhagen, Denmark, May 10-11. En ligne : http://www. fvm.dk/kundeupload/konferencer/organic_ food_farming/temaer/theme3.htm.

Bougherara D. (2003). L'écolabellisation : un instrument de préservation de l'environnement par le consommateur? Une application aux produits agroalimentaires. Thèse de doctorat en économie, Université de Bourgogne, Dijon, pp. 1-425.

Bougherara D., Grolleau G. (2004). L'écoétiquetage des produits est-il crédible? Proposition d'un cadre d'analyse. Revue d'économie régionale et urbaine, $\mathrm{n}^{\circ} 3$, pp. 369-390.

Busch L., Thiagarajan D., Hatanaka M., Bain C., Flores L. G., Frahm M. (2005). The Relationship of Third-Party Certification (TPC) to Sanitary/Phytosanitary (SPS) Measures and the International Agri-Food Trade: Final Report. Raise SPS Global Analytical Report \#9, Washington D.C., USAID.
Carriquiry M., Babcock B. A., Carbone R. (2003). Optimal quality assurance systems for agricultural outputs. Center for Agricultural and Rural Development (CARD) Publications, 03-wp328, Iowa State University.

Caswell J. A., Mojduszka E. M. (1996). Using informational labeling to influence the market for quality in food products. American Journal of Agricultural Economics, vol. 78, $\mathrm{n}^{\circ} 5$, pp. 1248-1253.

Coestier B., Marette S. (2004). Économie de la qualité. Paris, La Découverte, pp. 1-128.

Darby M. R., Karni E. (1973). Free competition and the optimal amount of fraud. Journal of Law and Economics, vol. 16, $\mathrm{n}^{\circ} 1$, pp. 67-88.

Davenport T., Beck J. (2000). Getting attention. Harvard Business Review, $\mathrm{n}^{\circ} 119$, pp. 118-126.

Deaton J. (2004). A theoretical framework for examining the role of third-party certifiers. Food Control, n ${ }^{\circ}$ 15, pp. 615-619.

De Bon H., Temple L., Malézieux E., Bendjebbar P., Fouilleux E., Silvie P. (2017). Organic agriculture in Africa: A source of innovation for agricultural development. Perspective, édition française, $\mathrm{n}^{\circ} 48$, pp. 1-4.

Dufeu I. (2015). Stratégie et viabilité des systèmes agroalimentaires alternatifs. Mémoire d'HDR, Laboratoire d'Économie et de Management de Nantes-Atlantique, Université de Nantes, $181 \mathrm{p}$.

EAC (2007). East African Organic Products Standard. Tech. rep., East African Community https://www.oecd.org/aidfortrade/ 47719232.pdf.

Engel S. (2006). Overcompliance, labeling, and lobbying: The case of credence goods. Environmental Modeling and Assessment, vol. 11, n ${ }^{\circ}$, pp. 115-130.

Erdem T., Swait J., Louviere J. (2002). The impact of brand credibility on consumer price sensitivity. International Journal of Research in Marketing, vol. 19, $\mathrm{n}^{\circ}$ 1, pp. 1-19. 
Feddersen T. J., Gilligan T. W. (2001). Saints and markets: Activists and the supply of credence goods. Journal of Economics \& Management Strategy, vol. 10, $\mathrm{n}^{\circ} 1$, pp. 149-171.

Figuié M., Mayer J. (2010). À qui se fier quand les aliments font peur? Labels, points de vente et décontamination symbolique au Vietnam. Working Papers MOISA 201004, UMR Marchés, Organisations, Institutions et Stratégies d'Acteurs : CIHEAMIAMM, CIRAD, INRA, Montpellier SupAgro - Montpellier, France.

Fischer C., Lyon T. P. (2014). Competing environmental labels. Journal of Economics \& Management Strategy, vol. 23, $\mathrm{n}^{\circ} 3$, pp. 692-716.

Giannakas K. (2002). Information Asymmetries and Consumption Decisions in Organic Food Product Markets. Canadian Journal of Agricultural Economics, vol. 50, $\mathrm{n}^{\circ} 1$, pp. 35-50.

Grolleau G., Caswell J. A. (2006). Interaction between food attributes in markets: the case of environmental labeling. Journal of agricultural and resource economics, vol. 31, $\mathrm{n}^{\circ} 3$, pp. 471-484.

Goldberger J. R. (2008). Non-governmental organizations, strategic bridge building, and the "scientization" of organic agriculture in Kenya. Agriculture and Human Values, vol. 25, $\mathrm{n}^{\circ}$ 2, pp. 271-289.

Hirschman A. O. (1970). Exit, voice, and loyalty: Responses to decline in firms, organizations, and states. Cambridge, Harvard University Press.

Hirschman A. O. (1974). Exit, voice, and loyalty: Further reflections and a survey of recent contributions. Social Science Information, vol. $13, \mathrm{n}^{\circ} 1$, pp. 7-26.

Hirschman A. O. (1982). Shifting involvements: Private interest and public action. Princeton, Princeton University Press.

IFOAM (2013). Consumer survey of attitudes and preferences towards organic products in East Africa. International Federation of Organic Agriculture Movements (IFOAM), Bonn.

Jahn G., Schramm M., Spiller A. (2005). The reliability of certification: Quality labels as a consumer policy tool. Journal of Consumer Policy, vol. 28, n 1 , pp. 53-73.

Källander I. (2008). Participatory guarantee systems-PGS. Stockholm, Swedish Society for Nature Conservation, pp. 1-25.

Khosla R. (2006). A participatory organic guarantee system for India. FAO Report, https:// www.ifoam.bio/sites/.../pgs_for_india_ final_report.pdf.

Lizzeri A. (1999). Information Revelation and Certification of Intermediaries. Rand Journal of Economics, vol. 30, $\mathrm{n}^{\circ} 2$, pp. 214-231.

Lupton S. (2005). Shared quality uncertainty and the introduction of indeterminate goods. Cambridge Journal of Economics, vol. 29, $\mathrm{n}^{\circ} 3$, pp. 399-421.

Lupton S. (2009). Incertitude sur la qualité. De l'asymétrie d'information à l'incertitude partagée. Note pour l'obtention de l'habilitation à diriger des recherches, Université Paris X-Nanterre, pp. 1-78.

Lupton S. (2015). Quality uncertainty in early economic thought. History of Political Economy, vol. 47, n 3, pp. 511-534.

Manning L., Baines R. N. (2004). Effective management of food safety and quality. British Food Journal, vol. 106, n 8-9, pp. 598-606.

May C. (2008). Petit guide des SPG, ou comment développer et faire fonctionner les Systèmes Participatifs de Garantie. International Federation of Organic Agriculture Movements (IFOAM), Bonn, pp. 1-68.

McCluskey J. (2000). A game theoretic approach to organic foods: An analysis of asymmetric information and policy. Agricultural and Resource Economics Review, vol. 29, n 1 , pp. 1-9.

Ndungu S. K. (2013). Consumer Survey of Attitudes And Preferences Towards Organic Products in East Africa. IFOAM Report. En ligne : https://www.ifoam.bio/sites/.../osea_ ii_consumer_survey_final.pdf.

Nelson P. (1970). Information and consumer behavior. The Journal of Political Economy, vol. 78, n 2, pp. 311-329.

Probst L., Houedjofonon E., Ayerakwa H. M., Haas, R. (2012). Will they buy it? The 
RECHERCHE

potential for marketing organic vegetables in the food vending sector to strengthen vegetable safety: A choice experiment study in three West African cities. Food Policy, vol. 37, n 3, pp. 296-308.

Raynaud E., Sauvée L. (2000). Signes collectifs de qualité et structures de gouvernance. Économie rurale, vol. 258, $\mathrm{n}^{\circ} 1$, pp. 101-112.

Roe B., Sheldon I. (2007). Credence good labeling: The efficiency and distributional implications of several policy approaches. American Journal of Agricultural Economics, vol. 89, n ${ }^{\circ}$, pp. 1020-1033.

Rundgren G. (2007). PGS in East Africa. IFOAM commissioned consultancy, pp. 1-46.

Sawyer E. (2004). The economic impacts of harmonizing organic standards internationally. Ph.D. thesis, University of Saskatchewan Saskatoon, pp. 1-288.

Tankam C. (2015). Analyse économique du développement des nouveaux marchés biologiques. Le cas des marchés biologiques domestiques au Kenya. Thèse de doctorat en économie, Université d'AuvergneClermont-Ferrand I, pp. 1-268.

Tankam C. (2017). L'organisation des marchés de producteurs de fruits et légumes biologiques à Nairobi, Kenya. Cahiers Agricultures, vol. 26, n 3, pp.1-7.

Tanner B. (2000). Independent assessment by third-party certification bodies. Food Control, $\mathrm{n}^{\circ} 11$, pp. 415-417.
Taylor A. (2006). Overview of the current state of organic agriculture in Kenya, Uganda and the United Republic of Tanzania and the opportunities for regional harmonization, Report for UNEP-UNCTAD Capacity Building Task Force on Trade, Environment and Development, United Nations, New York and Geneva, pp. 1-65. En ligne: https://unctad.org/en/Docs/ditcted200516_ en.pdf

Tirole J. (1986). Hierarchies and Bureaucracies: On the Role of Collusion in Organisations. Journal of Law, Economics and Organization, vol. 2, ${ }^{\circ}$ 2, pp. 181-214.

UNEP (2010). Organic Agriculture: Opportunities for Promoting Trade, Protecting the Environment and Reducing Poverty. Case studies from East Africa, pp. 1-59.

Valceschini E., Mazé A. (2000). La politique de la qualité agroalimentaire dans le contexte international. Économie rurale, vol. 258, $\mathrm{n}^{\circ} 1$, pp. 30-41.

Van den Akker, J. (2009). Convergence entre les Systèmes Participatifs de Garantie et les Systèmes de Contrôle Interne dans un projet pilote européen d'Ifoam. Innovations agronomiques, $\mathrm{n}^{\circ} 4$, pp. 441-446.

Willer H., Lernoud J., Huber B., Sahota A. (2018, February). The world of organic agriculture. BIOFACH Session 2018 conference, pp. 1-33.

Winfree J. A., McCluskey J. J. (2005). Collective reputation and quality. American Journal of Agricultural Economics, vol. 87, $\mathrm{n}^{\circ} 1$, pp. 206-213. 